\title{
Neutrophil-to-lymphocyte ratio as a predictor of postoperative morbidity in patients with distal cholangiocarcinoma
}

\author{
YUSUKE KUMAMOTO, TAKASHI KAIZU, HIROSHI TAJIMA, NOBUYUKI NISHIZAWA, \\ SHIGENORI EI, KAZUHARU IGARASHI and MASAHIKO WATANABE
}

Department of Surgery, Kitasato University School of Medicine, Sagamihara, Kanagawa 252-0374, Japan

Received May 18, 2018; Accepted August 6, 2018

DOI: $10.3892 /$ mco.2018.1698

\begin{abstract}
Systemic inflammatory-, immunological- and nutritional-based indices, such as the neutrophil-to-lymphocyte ratio (NLR), the prognostic nutritional index (PNI) and the Glasgow prognostic score (GPS), are drawing considerable research attention to predict the long-term prognosis of many types of cancer. Recently, these parameters have also been reported to be useful in predicting postoperative morbidity in several fields, including colorectal and otolaryngological cancer. However, while distal cholangiocarcinoma exhibits a high morbidity rate, its risk factors of morbidity have not yet been established. This is because previous studies have analyzed distal cholangiocarcinoma as periampullary tumors combined with pancreatic head cancer. Therefore, the aim of the present study was to investigate the application of the NLR, the PNI and the GPS in evaluating risk factors for postoperative morbidity in patients with distal cholangiocellular or ampullary carcinoma. Eighty-four patients who underwent pancreaticoduodenectomy (PD) for distal cholangiocellular or ampullary carcinoma at the Department of Surgery in Kitasato University Hospital between 2008 and 2016 were enrolled. Associations between perioperative characteristics (NLR, PNI and GPS) and postoperative complications
\end{abstract}

Correspondence to: Professor Yusuke Kumamoto, Department of Surgery, Kitasato University School of Medicine, 1-15-1 Kitasato, Minami-ku, Sagamihara, Kanagawa 252-0374, Japan

E-mail: kumamoto@kitasato-u.ac.jp

Abbreviations: AUC, area under the curve; BMI, body mass index; $\mathrm{CD}$, clavien-dindo; $\mathrm{CI}$, confidence interval; CRP, C-reactive protein; GPS, glasgow prognostic score; NLR, neutrophil-to-lymphocyte ratio; $\mathrm{OR}$, odds ratio; $\mathrm{PD}$, pancreaticoduodenectomy; $\mathrm{PNI}$, prognostic nutritional index; PP, pylorus-preserving; PPPD, pylorus-preserving pancreaticoduodenectomy; ROC, receiver operating characteristic; SSP, subtotal stomach-preserving; SSPPD, substomach-preserving pancreaticoduodenectomy; TNM, tumor-node-metastasis; UICC, International Union Against Cancer

Key words: body mass index, complication, distal cholangiocarcinoma, neutrophil-to-lymphocyte ratio, pancreaticoduodenectomy, postoperative morbidity
(Clavien-Dindo classification grade III or higher) were retrospectively analyzed. In the univariate analysis, neutrophil and lymphocyte counts, body mass index (BMI) and the NLR were associated with postoperative complications $(\mathrm{P}<0.05)$. In the multivariate analysis, BMI $\left[>23.0 \mathrm{~kg} / \mathrm{m}^{2}\right.$; odds ratio $(\mathrm{OR})$ : 3.80, 95.0\% confidence interval $(\mathrm{CI}): 1.35-11.83 ; \mathrm{P}=0.011]$ and the NLR (>2.0; OR: 6.77, 95.0\% CI: 2.44-21.13; P<0.001) were independent risk factors for postoperative complications. BMI and the NLR are valuable predictors of postoperative morbidity following PD in patients with distal cholangiocarcinoma. It would be beneficial to determine treatment strategies for distal cholangiocarcinoma based on the NLR to reduce postoperative complications.

\section{Introduction}

It is increasingly recognized that the prognosis of patients with cancer is influenced by not only the oncological characteristics of the tumor, but also the conditions of the host. The relationship between the inflammatory and immunonutritional status and the prognosis of patients with cancer has been reported in several studies (1-4) in recent years. A number of indicators, including the neutrophil-to-lymphocyte ratio (NLR), the prognostic nutritional index (PNI), and the Glasgow prognostic score (GPS), have been reported to be useful predictors of overall or disease-free survival in numerous types of cancers (1-6). The NLR was first shown to correlate with short-term outcome in patients in an intensive care unit by Zahorec (7) in 2001. Recently, other studies (8-11) have also shown that these parameters are good predictors of postoperative complications in patients with colorectal or otolaryngological cancer. Therefore, these indicators may be effective markers of short-term outcome in various fields.

Distal cholangiocarcinoma is associated with a high morbidity rate. The majority of patients with these tumors already show signs of liver dysfunction, jaundice, and malnutrition (e.g., hypoalbuminemia due to obstruction of the biliary duct) at the first visit to the hospital $(12,13)$. Moreover, some patients exhibit an inflammatory reaction, such as cholangitis, and require biliary drainage during the preoperative period. Therefore, the biochemical data of patients with distal cholangiocarcinoma are likely to show abnormal values to some extent before surgery.

The only curative treatment for distal cholangiocarcinoma is surgical resection, such as pancreaticoduodenectomy (PD), 
which is a highly invasive and complicated procedure. Although the mortality rate of PD has decreased satisfactorily to $5.0 \%$ in high volume centers, due to improvements in surgical techniques and perioperative management, the postoperative morbidity rate remains high, with values ranging from $30.0-65.0 \%(14,15)$. Several studies $(16-18)$ have reported risk factors for postoperative complications after PD. However, the patients recruited to these studies included those with distal cholangiocarcinoma, as well as, pancreatic head cancer. A recent study of a large cohort of patients with resected periampullary cancer (13) showed that patients with cholangiocarcinoma had a higher morbidity rate than those with pancreatic head cancer and that apparent biological differences existed between the 2 groups. Few studies have evaluated the risk of morbidity associated with only distal cholangiocarcinoma (19) and risk factors for postoperative complications after PD in patients with distal cholangiocarcinoma have not yet been established.

We hypothesized that systemic inflammatory, immunological, and nutritional status would influence the morbidity rate of patients with distal cholangiocarcinoma. The aim of this study was to determine the effectiveness of the NLR, the PNI, and the GPS in evaluating risk factors for postoperative complications after PD in patients with distal cholangiocarcinoma.

\section{Materials and methods}

Patients. We conducted a retrospective analysis of 84 patients who underwent PD for pathologically confirmed distal cholangiocarcinoma, including distal cholangiocellular and ampullary carcinoma, at Kitasato University Hospital (Kitasato, Japan) between January 2008 and December 2016. Patients who had undergone simultaneous hepatic resection were excluded. Clinical, surgical, and tumor characteristics, including age, sex, body mass index (BMI), biliary drainage, bile culture, laboratory data, operating times, blood loss and transfusion, International Union Against Cancer (UICC) tumor-node-metastasis (TNM) stage, and postoperative hospital stay, were collected and analyzed from the clinical database.

All patients underwent pylorus-preserving (PP), subtotal stomach-preserving (SSP), or conventional PD with regional lymph node dissection. Portal vein resection was performed when the tumor had invaded to achieve resection-free margins. Reconstruction was a modified Child's reconstruction. Pancreaticojejunostomy was performed according to the Kakita et al (20) method, as described previously (i.e., duct-to-mucosa anastomosis with a lost pancreatic stent and end-to-side anastomosis with 4 stitches of transpancreatic suture in all patients). Two closed drains were routinely inserted at the Winslow's space in front of the pancreaticojejunostomy. Drain management was as follows, the drains were removed on day 4 under condition that the amylase level of drain fluid were less than $4,000 \mathrm{U} / \mathrm{ml}$ or the volume of drain fluid were less than $30 \mathrm{ml}$. If this condition was not satisfied or drain fluid were infected, the drains were continued to drainage.

Postoperative complications. Postoperative complications, such as anastomotic leakage, postpancreatectomy hemorrhage, liver abscess, intraperitoneal abscess, allergic reaction, and pneumonia, were classified according to the Clavien-Dindo (CD) classification (21). Patients with CD classification grade III or higher postoperative complications were assigned to the major complications group, and the remainder assigned to the non-major complications group.

NLR, PNI, and GPS evaluation. Blood test results from the day immediately prior to surgery were used. The NLR was calculated as the absolute neutrophil count divided by the absolute lymphocyte count. The PNI was calculated as $10 \mathrm{x}$ albumin $+0.05 \mathrm{x}$ the lymphocyte count. The GPS was determined based on C-reactive protein (CRP) and albumin. Patients with elevated CRP $(>0.3 \mathrm{mg} / \mathrm{dl})$ and hypoalbuminemia $(<3.5 \mathrm{mg} / \mathrm{dl})$ were assigned a score of 2 . Patients with either one of these 2 biochemical outliers were assigned a score of 1 . Patients with neither of these biochemical outliers were assigned a score of $0(22)$.

Statistical analyses. Categorical variables were compared using the Chi-square test or Fisher's exact test. Continuous variables were compared using the Student's t-test or Mann-Whitney U test. Continuous variables were presented as the mean and standard deviation. Receiver operating characteristic (ROC) curve analysis was performed to determine the optimal cut-off values for several variables to predict postoperative complications. Variables associated with postoperative complications in the univariate analysis were included in the multivariate logistic regression analysis. Odds ratio (OR) and $95.0 \%$ confidence intervals (CIs) were used to quantify the strength of the association between predictors and morbidity. All statistical analyses were conducted using $\mathrm{JMP}^{\circledR}$ software (version 11.2.0; SAS Institute Inc., Cary, NC, USA). A $\mathrm{P}<0.05$ was considered statistically significant.

\section{Results}

In total, 84 patients were enrolled in the study. The characteristics of the patients are summarized in Table I. The mean age was $68.1 \pm 8.7$ years. Sixty-three patients were male and 21 patients were female. Fifty-seven patients had distal cholangiocellular carcinoma and 27 patients had ampullary carcinoma. Seventy-four patients underwent PPPD, 7 patients underwent SSPPD, and 3 patients underwent conventional PD. Thirty-nine patients (46.4\%) experienced postoperative complications of CD classification grade III or higher. The most common postoperative complication was anastomotic leakage $(n=31 ; 36.9 \%)$ [pancreaticojejunostomy leakage $(n=27)$, choledochojejunostomy leakage $(n=2)$, and duodenojejunostomy leakage $(n=2)]$. Other complications included bleeding $(n=2)$, intraperitoneal abscess $(n=2)$, pneumonia $(\mathrm{n}=2)$, liver abscess $(\mathrm{n}=1)$, and anaphylactic shock $(\mathrm{n}=1)$.

First of all, we compared patients with ampullary carcinoma and those with distal cholangiocelluler carcinoma. The clinical characteristics of each group are summarized in Table II. Biliary drainage rate was over $90 \%$ in both groups. Patients with distal cholangiocellular carcinoma showed high frequency of bile culture positive (88\%) and slightly high CRP $(1.44 \pm 2.14)$ rather 
Table I. Patient characteristics $(n=84)$.

\begin{tabular}{ll}
\hline Patient characteristic & \multicolumn{1}{c}{ Data } \\
\hline Age (years) & $68.1 \pm 8.7$ \\
Sex & Male, 63 $(75 \%)$ \\
& Female, 21 (25\%) \\
Diagnosis & Ampullary carcinoma, 27 (32.1\%) \\
& Distal cholangiocellular carcinoma, \\
& $57(67.9 \%)$ \\
Operative procedure & PPPD, 74 (88.1\%) \\
& SSPPD, 7 (8.3\%) \\
& PD, 3 (3.6\%) \\
Complication ( $\geq$ CDIII) & Anastomotic leakage, 31 (36.9\%) \\
& Intra-abdominal abscess, 2(2.4\%) \\
& Bleeding, 2 (2.4\%) \\
& Pneumonia, 2 (2.4\%) \\
& Liver abscess, 1 (1.2\%) \\
& Anaphylactic shock, 1 (1.2\%)
\end{tabular}

CD, Clavien-Dindo; PD, pancreaticoduodenectomy; PPPD, pylorus-preserving pancreaticoduodenectomy; SSPPD, subtotal stomach-preserving pancreaticoduodenectomy.

than ampullary carcinoma (70\% and $0.60 \pm 0.64$, respectively), but with no statistically significant difference $(\mathrm{P}=0.06$ and 0.051 , respectively). Other preoperative biochemical variables and operative parameters including operative times, blood loss volumes, the incidence of transfusion or portal vein resection were comparable between the 2 groups. There was no significant difference in UICC TNM stage, NLR and GPS, however patients with ampullary carcinoma showed higher level of PNI rather than distal cholangiocellular carcinoma with statistically significant difference. The complication rate over grade III in $\mathrm{CD}$ classification and postoperative hospital stays were comparable between the 2 groups.

Next, the patients were divided into 2 groups depending on the presence of complication or not for evaluating risk factor of postoperative complication. The 39 patients with postoperative complications of CD classification grade III or higher were assigned to the major complications group. The remaining 45 patients were assigned to the non-major complications group. The clinical characteristics of each group are summarized in Table III. Preoperative biochemical variables, including platelet count and total bilirubin, alkaline phosphatase, gamma-glutamyltransferase, albumin, and CRP levels, were comparable between the 2 groups. There were no significant differences in mean operative times, blood loss volumes, the incidence of blood transfusions or portal vein resection, UICC TNM stage, the frequencies of biliary drainage and bile culture positive. The major complications group had a significantly higher neutrophil count $(\mathrm{P}<0.05)$, NLR $(\mathrm{P}<0.01)$, and BMI $(\mathrm{P}<0.01)$ than the non-major complications group. In contrast, the lymphocyte count was significantly lower in the major complications group than in the non-major complications group $(\mathrm{P}<0.05)$. Other indicators, such as the PNI and the GPS, did not differ significantly between the 2 groups. Understandably, the postoperative hospital stay was statistically long in the major complications group rather than in the non-major complications group.

Variables for which significant differences were identified in the univariate analysis were further analyzed using ROC curve analysis to determine the optimal cut-off values. The optimal cut-off value for the NLR was 2.0 [area under curve (AUC), 0.72; Fig. 1A]. The optimal cut-off value for the BMI was $23.0 \mathrm{~kg} / \mathrm{m}^{2}$ (AUC, 0.67; Fig. 1B). The optimal cut-off values for the neutrophil count and lymphocyte count were 2,727 (AUC, 0.67) and 1,870 (AUC, 0.59), respectively. These parameters were included in the multivariate logistic regression analysis. A BMI of $>23.0 \mathrm{~kg} / \mathrm{m}^{2}$ (OR: $3.83,95.0 \%$ CI: 1.35-11.83; $\mathrm{P}=0.011$ ) and a NLR of $>2.0$ (OR: 6.77, 95.0\% CI: 2.44-21.13; $\mathrm{P}<0.001$ ) were independent risk factors for major postoperative complications (Table IV).

\section{Discussion}

The present study highlights the usefulness of the NLR in predicting postoperative major complications after PD in patients with distal cholangiocarcinoma, providing surgeons with important information. Surgeons sometimes confront the situation that patients with biliary carcinoma experience acute cholangitis immediately prior to surgery. Acute cholangitis activates inflammatory responses, whilst suppressing immunological and nutritional responses, resulting in elevated neutrophil counts and CRP levels and reduced lymphocyte counts and albumin levels. In such cases, antibiotic treatment or biliary drainage (stenting) were performed. However, we do not know how long we should wait for the patient's condition to improve. Our findings suggest that surgeons may be able to determine treatment strategies based on the NLR.

Over the past few decades, many studies (14-18,23-25) have reported that the risk factors for postoperative complications after PD include a soft pancreatic texture, a small pancreatic duct diameter, a high BMI, preoperative biliary drainage, operative blood loss, and sarcopenia. However, the patients enrolled in these studies have included a mixture of those with distal cholangiocarcinoma and pancreatic head cancer. Andrianello et al (13) reported that the postoperative morbidity rate was significantly higher in patients with distal cholangiocarcinoma than in those with pancreatic ductal adenocarcinoma (75.9\% vs. $52.1 \%$, respectively; $\mathrm{P}<0.01$ ). Preoperative variables [e.g., the incidence of jaundice $(94.4 \%$ vs. $72.0 \%$, respectively; $\mathrm{P}<0.01)$ and biliary stent insertion ( $79.6 \%$ vs. $54.4 \%$, respectively; $\mathrm{P}<0.01)$ ] also differed significantly between the 2 groups. In this study, we compared patients with ampullary carcinoma and those with distal cholangiocellular carcinoma. Both groups showed the same frequencies of biliary drainage $(93 \%$ vs. $98 \%$, respectively; $\mathrm{P}=0.212$ ). Concerning bile juice infection, slightly higher incidence was observed in patients with distal cholangiocellular carcinoma (88\%) compared to patients with ampullary carcinoma (70\%), however the frequency of postoperative complications was observed equally between 2 groups (44\% vs. $52 \%$; $\mathrm{P}=0.493$ ).

Finally, a high NLR and a high BMI were extracted as independent risk factors for postoperative complications after PD for 
Table II. Characteristics of patients with ampullary carcinoma and patients with distal cholangiocellular carcinoma.

\begin{tabular}{|c|c|c|c|}
\hline Variable & $\begin{array}{c}\text { Ampullary } \\
\text { carcinoma }(n=27)\end{array}$ & $\begin{array}{l}\text { Distal cholangiocellular } \\
\text { carcinoma }(n=57)\end{array}$ & $\mathrm{P}$-value \\
\hline Age (years) & $65.8 \pm 10.3$ & $69.2 \pm 7.7$ & 0.092 \\
\hline $\operatorname{Sex}(M / F)$ & $18 / 9$ & $45 / 12$ & 0.704 \\
\hline Total bilirubin (mg/dl) & $0.87 \pm 0.62$ & $1.02 \pm 0.61$ & 0.326 \\
\hline $\operatorname{ALP}(\mathrm{U} / \mathrm{l})$ & $448.6 \pm 65.9$ & $546.6 \pm 45.3$ & 0.224 \\
\hline GTP (U/1) & $149.8 \pm 53.1$ & $267.7 \pm 6.6$ & 0.071 \\
\hline Platelets, $\mathrm{x} 10^{4} / \mu 1$ & $26.2 \pm 10.3$ & $24.6 \pm 6.6$ & 0.385 \\
\hline Neutrophils/ $\mu \mathrm{l}$ & $3,261 \pm 1,228$ & $3,504 \pm 1,269$ & 0.411 \\
\hline Lymphocytes/ $\mu 1$ & $1,558 \pm 552$ & $1,437 \pm 479$ & 0.308 \\
\hline Albumin (g/dl) & $3.79 \pm 0.07$ & $3.62 \pm 0.05$ & 0.066 \\
\hline CRP (mg/dl) & $0.60 \pm 0.64$ & $1.44 \pm 2.14$ & 0.051 \\
\hline BMI & $21.94 \pm 3.35$ & $22.43 \pm 3.39$ & 0.541 \\
\hline Biliary drainage $(+)$ & $25(93 \%)$ & $56(98 \%)$ & 0.212 \\
\hline Bile culture positive & $19(70 \%)$ & $50(88 \%)$ & 0.060 \\
\hline Operation time (min) & $505 \pm 130$ & $537 \pm 117$ & 0.263 \\
\hline Blood loss (ml) & $1,293 \pm 1,142$ & $1,196 \pm 899$ & 0.682 \\
\hline Transfusion (+) & $7(26 \%)$ & $10(18 \%)$ & 0.379 \\
\hline PV resection (+) & $1(4 \%)$ & $6(11 \%)$ & 0.259 \\
\hline Stage & & & 0.195 \\
\hline IA & 7 & 5 & \\
\hline IB & 3 & 8 & \\
\hline IIA & 4 & 18 & \\
\hline IIB & 12 & 24 & \\
\hline III & 0 & 0 & \\
\hline IV & 1 & 1 & \\
\hline NLR & $2.40 \pm 1.60$ & $2.68 \pm 1.32$ & 0.392 \\
\hline PNI & $45.64 \pm 4.82$ & $43.36 \pm 4.39$ & 0.034 \\
\hline GPS & & & 0.388 \\
\hline 0 & 16 & 26 & \\
\hline 1 & 9 & 22 & \\
\hline 2 & 2 & 9 & \\
\hline Complication ( $\geq$ CDIII) & $14(52 \%)$ & $25(44 \%)$ & 0.493 \\
\hline Postoperative hospital stay (days) & $25.4 \pm 17.7$ & $26.9 \pm 20.7$ & 0.743 \\
\hline
\end{tabular}

M, male; F, female; ALP, alkaline phosphatase; BMI, body mass index; CD, Clavien-Dindo classification; CRP, C-reactive protein; GPS, Glasgow prognostic score; $\gamma$ GTP, $\gamma$-glutamyl transferase; NLR, neutrophil-to-lymphocyte ratio; PNI, prognostic nutritional index; PV, portal vein.

distal cholangiocarcinoma. Yamashita et al (26) reported that a BMI of $\geq 25.0 \mathrm{~kg} / \mathrm{m}^{2}$ is an independent risk factor for clinically relevant pancreatic fistulas after PD for pancreatic ductal adenocarcinoma. The authors speculate that this may be because the pancreas of patients with a high BMI contains a large fatty component, which results in a fragile parenchyma (so called 'soft pancreas') (27-29). It seems acceptable that the BMI threshold in our study was lower than that of the Yamashita et al (26) study because pancreatic exocrine function is well preserved in patients with cholangiocarcinoma compared to those with pancreatic ductal adenocarcinoma (30).

In the last decade, the NLR has emerged as an attractive proxy to characterize systemic inflammatory status. Several studies $(1,3,31)$ have suggested that a high NLR is a poor prognostic marker because neutrophilia accelerates tumor vessel angiogenesis and lymphopenia diminishes immune protection against tumor invasion. Therefore, a high NLR is associated with favorable tumor conditions. However, the reason why the NLR is concerning for postoperative complications may be related to quite different mechanisms. The mechanisms underlying associations between systemic inflammation and postoperative complications remain to be elucidated. Josse et al (10) reported that a high NLR of $>2.3$ correlated with postoperative complications, specifically anastomotic leakage and not infections, in patients with colorectal cancer. Kudo et al (32) reported an association between the perioperative NLR and postoperative complications, specifically infections, in patients with colorectal cancer. Considering 
Table III. Comparison between clinical characteristics and perioperative outcomes between the major and non-major complications groups.

\begin{tabular}{|c|c|c|c|}
\hline Variable & $\begin{array}{l}\text { Major complications } \\
\text { group }(n=39)\end{array}$ & $\begin{array}{l}\text { Non-major complications } \\
\text { group }(n=45)\end{array}$ & P-value \\
\hline Age (years) & $69.2 \pm 8.5$ & $67.3 \pm 8.9$ & 0.325 \\
\hline $\operatorname{Sex}(M / F)$ & $30 / 9$ & $33 / 12$ & 0.704 \\
\hline Total bilirubin (mg/dl) & $0.86 \pm 0.10$ & $1.06 \pm 0.65$ & 0.131 \\
\hline $\operatorname{ALP}(\mathrm{U} / \mathrm{l})$ & $491.7 \pm 55.2$ & $535.4 \pm 51.4$ & 0.563 \\
\hline GTP (U/l) & $232.5 \pm 45.1$ & $227.5 \pm 42.0$ & 0.935 \\
\hline Platelets, $\times 10^{4} / \mu 1$ & $24.1 \pm 7.5$ & $26.0 \pm 8.4$ & 0.293 \\
\hline Neutrophils/ $\mu 1$ & $3,758 \pm 1,138$ & $3,138 \pm 1,290$ & 0.023 \\
\hline Lymphocytes $/ \mu 1$ & $1,353 \pm 375$ & $1,582 \pm 576$ & 0.037 \\
\hline Albumin $(\mathrm{g} / \mathrm{dl})$ & $3.68 \pm 0.06$ & $3.67 \pm 0.06$ & 0.905 \\
\hline $\mathrm{CRP}(\mathrm{mg} / \mathrm{dl})$ & $1.15 \pm 1.57$ & $1.18 \pm 2.06$ & 0.943 \\
\hline BMI & $23.3 \pm 3.67$ & $21.35 \pm 2.80$ & 0.007 \\
\hline Biliary drainage (+) & $38(97 \%)$ & $43(96 \%)$ & 0.639 \\
\hline Bile culture positive & $33(85 \%)$ & $36(80 \%)$ & 0.580 \\
\hline Operation time (min) & $535 \pm 130$ & $519 \pm 115$ & 0.553 \\
\hline Blood loss (ml) & $1,359 \pm 1,201$ & $1,119 \pm 743$ & 0.282 \\
\hline Transfusion (+) & $11(28 \%)$ & $6(13 \%)$ & 0.090 \\
\hline PV resection (+) & $1(3 \%)$ & $6(13 \%)$ & 0.060 \\
\hline Stage & & & 0.327 \\
\hline IA & 5 & 7 & \\
\hline IB & 6 & 5 & \\
\hline IIA & 13 & 9 & \\
\hline IIB & 15 & 21 & \\
\hline III & 0 & 0 & \\
\hline IV & 0 & 2 & \\
\hline NLR & $3.04 \pm 1.46$ & $2.21 \pm 1.26$ & 0.007 \\
\hline PNI & $43.54 \pm 4.82$ & $44.58 \pm 4.46$ & 0.306 \\
\hline GPS & & & 0.846 \\
\hline 0 & 19 & 23 & \\
\hline 1 & 14 & 17 & \\
\hline 2 & 6 & 5 & \\
\hline Postoperative hospital stays (days) & $38.3 \pm 21.9$ & $16.1 \pm 9.1$ & $<0.0001$ \\
\hline
\end{tabular}

M, male; F, female; ALP, alkaline phosphatase; BMI, body mass index; CRP, C-reactive protein; GPS, Glasgow prognostic score; $\gamma \mathrm{GTP}$, $\gamma$-glutamyl transferase; NLR, neutrophil-to-lymphocyte ratio; PNI, prognostic nutritional index; PV, portal vein.

these findings, one potential explanation is that subclinical inflammation (e.g., latent cholangitis) may be exaggerated by surgery in a second attack. Excessive postoperative systemic inflammatory response syndrome causes a cytokine storm that results in microcirculatory disturbances in whole organs. In this study, the most common complication was anastomotic leakage. Nakanishi et al (33) reported that systemic inflammatory responses modified endothelial function. An inability of the endothelium to produce nitric oxide and prostacyclin could result in depletion of the vasodilator and antithrombotic properties of the vascular endothelium. Yao et al (34) also reported that a high NLR was associated with vascular thrombosis. Thus, a high NLR seems to drive microvascular impairment, which is unfavorable for wound healing.
A value of 2.0, as determined by ROC curve analysis, was selected as the threshold for the NLR in this study. This value was relatively low compared to that reported in the literature (5.0) (1). We speculate that this may be because systemic inflammatory responses need to be sustained for a prolonged period and with sufficient power to influence prognosis compared to postoperative complications. Other studies $(8,29)$ have reported thresholds of 2.3 for postoperative complications in colorectal surgery. It is reasonable that the threshold is 2.0 for PD and 2.3 for colorectal surgery because PD is a more invasive procedure than colorectal surgery.

A high preoperative NLR of $>2.0$ is a novel indicator for identifying patients at high risk of postoperative complications 

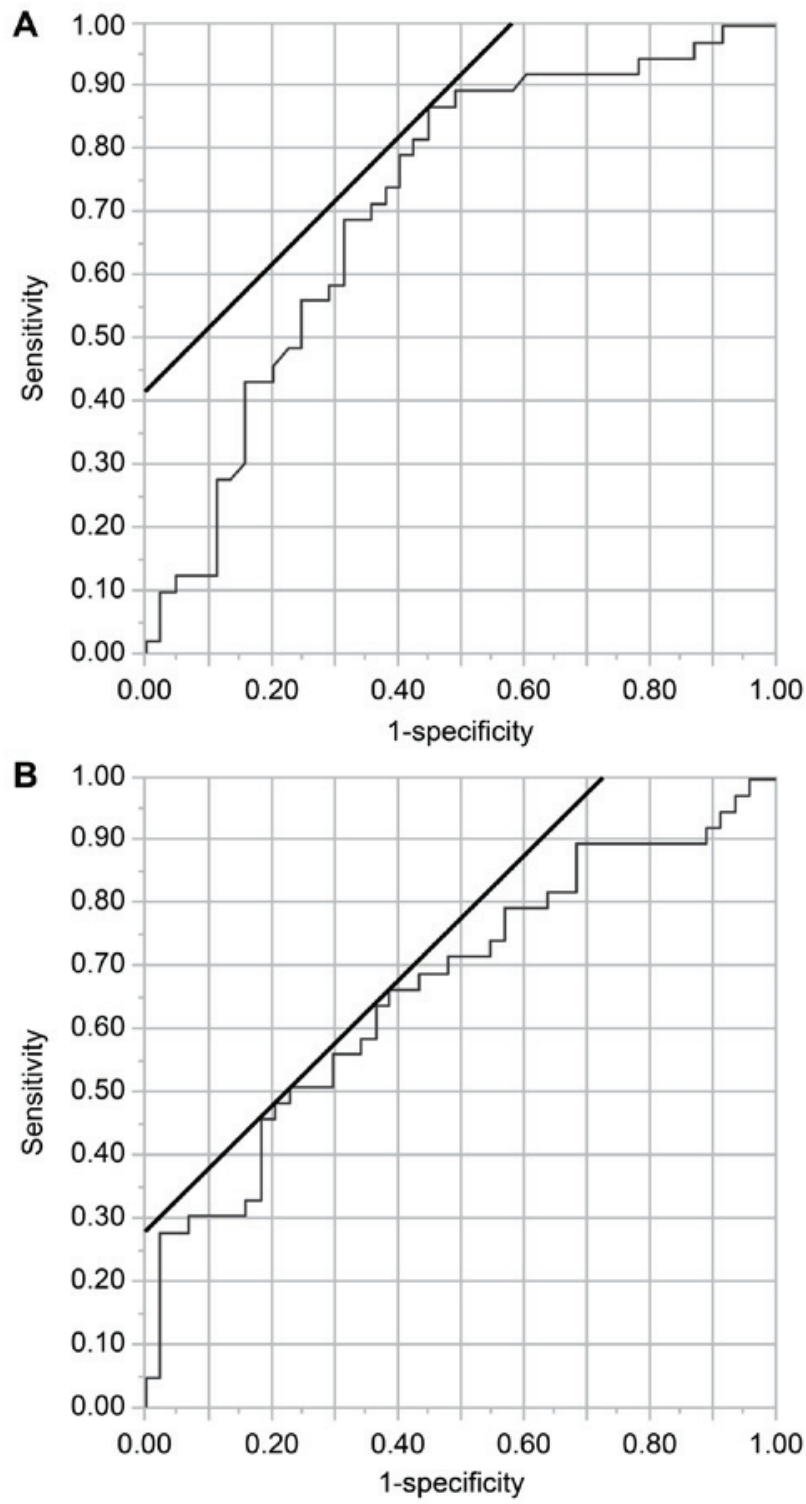

Figure 1. Receiver operating characteristic curve analysis of (A) the neutrophil-to-lymphocyte ratio and (B) the body mass index. The cut-off values were defined as those which corresponded to the points situated furthest away from the reference line.

after PD. As the NLR can be calculated from routine blood test results, it is cost effective and a convenient biomarker for surgeons to determine the best time to operate or to select different strategies for positive surgical outcomes.

This study has several limitations associated with its single-center retrospective design, limited sample size, and 8-year enrollment period. Although the perioperative management and surgical procedures have remained relatively constant, the skills and experiences of the surgeons were not included in the analysis. As cholangiocarcinoma is a rare disease, large multicenter studies would be desirable to validate our findings in a prospective manner.

In conclusion, a high NLR and a high BMI are risk factors of morbidity after PD in patients with distal cholangiocarcinoma. To our knowledge, this is the first study to report the effectiveness of the NLR for predicting postoperative complications in the pancreato-biliary field. Based on our findings, aggressive therapeutic intervention and/or waiting for a reduction in the
Table IV. Multivariate logistic regression analysis of the influence of clinical parameters on postoperative complications.

\begin{tabular}{lllr}
\hline Variable & OR & $95 \%$ CI & P-value \\
\hline Neutrophil $>2,727$ & 2.08 & $0.68-6.49$ & 0.201 \\
Lymphocyte $<1,870$ & 2.70 & $0.44-22.81$ & 0.290 \\
NLR $>2.0$ & 6.77 & $2.44-21.13$ & $<0.001$ \\
BMI $>23.0 \mathrm{~kg} / \mathrm{m}^{2}$ & 3.83 & $1.35-11.83$ & 0.011 \\
\hline
\end{tabular}

BMI, body mass index; CI, confidence interval; NLR, neutrophil-tolymphocyte ratio; OR, odds ratio.

NLR are recommended for reducing morbidity in patients with distal cholangiocarcinoma and a high preoperative NLR.

\section{Acknowledgements}

Not applicable.

\section{Funding}

No funding was received.

\section{Availability of data and materials}

The datasets used and/or analyzed during the current study are available from the corresponding author on reasonable request.

\section{Authors' contributions}

YK designed this study and wrote the whole draft of the manuscript. TK and HT contributed to the preparation of the clinical data. NN and SE contributed to analysis the data. KI contributed to the preparation of documents for the local ethics committee and search literatures. MW supervised the study. All authors read and approved the final manuscript.

\section{Ethics approval and consent to participate}

The study was approved by the Ethics Committee of Kitasato University School of Medicine (Kitasato, Japan). Research was conducted in accordance with the 1964 Declaration of Helsinki and its later amendments.

\section{Patient consent for publication}

Not applicable

\section{Competing interests}

The authors declare that they have no competing interests.

\section{References}

1. Paramanathan A, Saxena A and Morris DL: A systematic review and meta-analysis on the impact of pre-operative neutrophil lymphocyte ratio on long term outcomes after curative intent resection of solid tumours. Surg Oncol 23: 31-39, 2014. 
2. Nozoe T, Ninomiya M, Maeda T, Matsukuma A, Nakashima $H$ and Ezaki T: Prognostic nutritional index: A tool to predict the biological aggressiveness of gastric carcinoma. Surg Today 40: 440-443, 2010

3. Absenger G, Szkandera J, Stotz M, Postlmayr U, Pichler M, Ress AL, Schaberl-Moser R, Loibner H, Samonigg H and Gerger A: Preoperative neutrophil-to-lymphocyte ratio predicts clinical outcome in patients with stage II and III colon cancer. Anticancer Res 33: 4591-4594, 2013.

4. Zhang P, Xi M, Li QQ, He LR, Liu SL, Zhao L, Shen JX and Liu MZ: The modified glasgow prognostic score is an independent prognostic factor in patients with inoperable thoracic esophageal squamous cell carcinoma undergoing chemoradiotherapy. J Cancer 5: 689-695, 2014.

5. He C, Mao Y, Lao X, Li S and Lin X: Neutrophil-to-lymphocyte ratio predicts overall survival of patients with combined hepatocellular cholangiocarcinoma. Oncol Lett 15: 4262-4268, 2018.

6. Han F, Shang X, Wan F, Liu Z, Tian W, Wang D, Liu Y, Wang Y, Zhang B and Ju Y: Clinical value of the preoperative neutrophil-to-lymphocyte ratio and red blood cell distribution width in patients with colorectal carcinoma. Oncol Lett 15: 3339-3349, 2018

7. Zahorec R: Ratio of neutrophil to lymphocyte counts-rapid and simple parameter of systemic inflammation and stress in clinically ill. Bratisl Lek Listy 102: 5-14, 2001 (In English, Slovak).

8. Nozoe T, Kimura Y, Ishida M, Saeki H, Korenaga D and Sugimachi K: Correlation of pre-operative nutritional condition with post-operative complications in surgical treatment for oesophageal carcinoma. Eur J Surg Oncol 28: 396-400, 2002.

9. Maruyama Y, Inoue K, Mori K, Gorai K, Shimamoto R, Onitsuka T, Iguchi $\mathrm{H}$, Okazaki $\mathrm{M}$ and Nakagawa $\mathrm{M}$ : Neutrophil-lymphocyte ratio and platelet-lymphocyte ratio as predictors of wound healing failure in head and neck reconstruction. Acta Otolaryngol 137: 106-110, 2017.

10. Josse JM, Cleghorn MC, Ramji KM, Jiang H, Elnahas A, Jackson TD, Okrainec A and Quereshy FA: The neutrophil-to-lymphocyte ratio predicts major perioperative complications in patients undergoing colorectal surgery. Colorectal Dis 18: O236-O242, 2016.

11. Kang WM, Zhu CZ, Yang XX, Yu JC, Ma ZQ, Ye X, Li K and Liu D: Application of the Onodera prognostic nutrition index and neutrophil-to-lymphocyte ratio in risk evaluation of postoperative complications in Crohn's disease. Sci Rep 7: 8481, 2017.

12. El Nakeeb A, Askar W, Atef E, Hanafy EE, Sultan AM, Salah T, Shehta A, Sorogy ME, Hamdy E, Hemly ME, et al: Trends and outcomes of pancreaticoduodenectomy for periampullary tumors: A 25-year single-center study of 1000 consecutive cases. World J Gastroenterol 23: 7025-7036, 2017.

13. Andrianello S, Marchegiani G, Malleo G, Rusev BC, Scarpa A, Bonamini D, Maggino L, Bassi C and Salvia R: Over 700 whipples for pancreaticobiliary malignancies: Postoperative morbidity is an additional negative prognostic factor for distal bile duct cancer. J Gastrointest Surg 21: 527-533, 2017.

14. Kawai M, Tani M, Hirono S, Ina S, Miyazawa M and Yamaue H: How do we predict the clinically relevant pancreatic fistula after pancreaticoduodenectomy?-an analysis in 244 consecutive patients. World J Surg 33: 2670-2678, 2009.

15. Yamamoto Y, Sakamoto Y, Nara S, Esaki M, Shimada K and Kosuge T: A preoperative predictive scoring system for postoperative pancreatic fistula after pancreaticoduodenectomy. World J Surg 35: 2747-2755, 2011

16. Yamane H, Abe T, Amano H, Hanada K, Minami T, Kobayashi T, Fukuda T, Yonehara S, Nakahara M, Ohdan H and Noriyuki T: Visceral adipose tissue and skeletal muscle index distribution predicts severe pancreatic fistula development after pancreaticoduodenectomy. Anticancer Res 38: 1061-1066, 2018.

17. Mungroop TH, van Rijssen LB, van Klaveren D, Smits FJ, van Woerden V, Linnemann RJ, de Pastena M, Klompmaker S, Marchegiani G, Ecker BL, et al: Alternative fistula risk score for pancreatoduodenectomy (a-FRS): Design and international external validation. Ann Surg: Dec 12, 2017 (Epub ahead of print).

18. Gagnière J, Abjean A, Franz M, Aumont O, Pereira B, Dupré A Veziant J, Le Roy B, Boyer L, Pezet D and Buc E: A normal preoperative lipase serum level is an easy and objective risk factor of pancreatic fistula after pancreaticoduodenectomy. Pancreas 46: 1133-1140, 2017.
19. Uemetsu S, Wakiya T, Ishido K, Kudo D, Kimura N, Miura T, Toyoki Y and Hakamada K: Effect of sarcopenia on the outcomes after pancreaticoduodenectomy for distal cholangiocarcinoma. ANZ J Surg: Feb 1, 2018 (Epub ahead of print). doi 10.1111/ans.14304.

20. Kakita A, Yoshida M and Takahashi T: History of pancreaticojejunostomy in pancreaticoduodenectomy: Development of a more reliable anastomosis technique. J Hepatobiliary Pancreat Surg 8 : 230-237, 2001

21. Dindo D, Demartines N and Clavien PA: Classification of surgical complications: A new proposal with evaluation in a cohort of 6336 patients and results of a survey. Ann Surg 240: 205-213, 2004

22. Proctor MJ, Morrison DS, Talwar D, Balmer SM, O'Reilly DS, Foulis AK, Horgan PG and McMillan DC: An inflammation-based prognostic score (mGPS) predicts cancer survival independent of tumour site: A glasgow inflammation outcome study. Br J Cancer 104: 726-734, 2011.

23. Fujii T, Yamada S, Suenaga M, Kanda M, Takami H, Sugimoto H, Nomoto S, Nakao A and Kodera Y: Preoperative internal biliary drainage increases the risk of bile juice infection and pancreatic fistula after pancreatoduodenectomy: A prospective observational study. Pancreas 44: 465-470, 2015.

24. Fang $\mathrm{CH}$, Chen QS, Yang J, Xiang F, Fang ZS and Zhu W: Body mass index and stump morphology predict an increased incidence of pancreatic fistula after pancreaticoduodenectomy. World J Surg 40: 1467-1476, 2016.

25. Nishida Y, Kato Y, Kudo M, Aizawa H, Okubo S, Takahashi D, Nakayama Y, Kitaguchi K, Gotohda N, Takahashi S and Konishi M: Preoperative sarcopenia strongly influences the risk of postoperative pancreatic fistula formation after pancreaticoduodenectomy. J Gastrointest Surg 20: 1586-1594, 2016.

26. Yamashita Y, Yoshizume T, Fukuzawa K, Nishizaki T, Tsujita E, Kajiyama K, Soejima Y, Yamagata M, Yamamoto K, Adachi E, et al: Surgical results of pancreaticoduodenectomy for pancreatic ductal adenocarcinoma: A multi-institutional retrospective study of 174 patients. Anticancer Res 36: 2407-2412, 2016.

27. Gaujoux S, Cortes A, Couvelard A, Noullet S, Clavel L, Rebours V, Lévy P, Sauvanet A, Ruszniewski P and Belghiti J: Fatty pancreas and increased body mass index are risk factors of pancreatic fistula after pancreaticoduodenectomy. Surgery 148: 15-23, 2010.

28. Rosso E, Casnedi S, Pessaux P, Oussoultzoglou E, Panaro F, Mahfud M, Jaeck D and Bachellier P: The role of 'fatty pancreas' and of BMI in the occurrence of pancreatic fistula after pancreaticoduodenectomy. J Gastrointest Surg 13: 1845-1851, 2009.

29. Olsen TS: Lipomatosis of the pancreas in autopsy material and its relation to age and overweight. Acta Pathol Microbiol Scand A 86A: 367-373, 1978

30. Callery MP, Pratt WB, Kent TS, Chaikof EL and Vollmer CM Jr: A prospectively validated clinical risk score accurately predicts pancreatic fistula after pancreatoduodenectomy. J Am Coll Surg 216: 1-14, 2013.

31. Walsh SR, Cook EJ, Goulder F, Justin TA and Keeling NJ: Neutrophil-lymphocyte ratio as a prognostic factor in colorectal cancer. J Surg Oncol 91: 181-184, 2005.

32. Kubo T, Ono S, Ueno H, Shinto E, Yamamoto J and Hase K: Impact of the perioperative neutrophil-to-lymphocyte ratio on the long-term survival following an elective resection of colorectal carcinoma. Int J Colorectal Dis 29: 1091-1099, 2014.

33. Nakanishi N, Sato M, Shirai K, Suzuki K and Tatara K: White blood cell count as a risk factor for hypertension; a study of Japanese male office workers. J Hypertens 20: 851-857, 2002.

34. Yao C, Zhang Z, Yao Y, Xu X, Jiang Q and Shi D: Predictive value of neutrophil to lymphocyte ratio and platelet to lymphocy te ratio for acute deep vein thrombosis after total joint arthroplasty: A retrospective study. J Orthop Surg Res 13: 40, 2018.

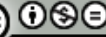

This work is licensed under a Creative Commons Attribution-NonCommercial-NoDerivatives 4.0 International (CC BY-NC-ND 4.0) License. 